\title{
La Responsabilidad Social Empresarial, una mirada a su aplicación en el sector transporte público automotor
}

\author{
A Look at the Application of Corporate Social Responsibility \\ to Public Transportation
}

Recibido: 31/10/2015 • Aprobado: 07/12/2015 • Página inicial: 77 - Página final: 99

\author{
Yunieth Delgado Palacio* \\ Natalia Herrera Cardona** \\ Carlos Andrés Gallón Ruiz*** \\ Martha Cecilia Álvarez Osorio**** \\ Lina María Muñoz Osorio*****
}

Resumen: la Responsabilidad Social Empresarial, RSE, es uno de los conceptos modernos que ha adquirido mayor relevancia en los últimos años en el mundo, en tanto implica un compromiso de las organizaciones y empresas para el bienestar común. Este artículo, resultado de investigación, parte por identificar las prácticas de RSE que aplica el sector transporte público automotor en el Valle de Aburrá, conforme con las tres dimensiones de la Guía Global Reporting Initiative (GRI): económico, social y ambiental; para lo cual se identificaron todas las empresas de transporte (buses) dedicadas a esta actividad y se evaluaron las prácticas de RSE que aplican, en concordancia con la guía.

Palabras clave: RSE, transporte público, GRI, prácticas de responsabilidad social empresarial, sostenibilidad.

\begin{abstract}
Corporate Social Responsibility (CSR) is one of the modern concepts that has gained the most relevance in the world in recent years, since it implies a commitment from organizations and corporations for common welfare. This paper resulted from research. It begins by identifying CSR practices that public transportation applies in Valle de Aburrá according to the three Global Reporting Initiative (GRI) dimensions: economic, social, and environmental. To this end, all transportation companies (buses) were identified, and the CSR practices they apply were evaluated as per the GRI.
\end{abstract}

Keywords: Corporate social responsibility, public transportation, Global Reporting Initiative (GRI), corporate social responsibility guidelines, sustainability.

JEL: M14

* Contadora Pública de la Universidad de Antioquia, Medellín - Colombia. yunieth@hotmail.com

** Contadora Pública de la Universidad de Antioquia, Medellín-Colombia. Natalia-he-ca@hotmail.com

*** Contador Público de la Universidad de Antioquia, Medellín - Colombia. carlosgallonr@hotmail.com

**** MSc. en Administración, Contadora Pública y docente vinculada al programa de Contaduría Pública de la Universidad de Antioquia, Medellín - Colombia. mcalvarezosorio@gmail.com

***** MSc. en Ciencias Contables, Contadora Pública y docente de tiempo completo del programa de Contaduría Pública de la Universidad de Antioquia, Medellín - Colombia.1mmunozo@gmail.com 


\section{Contexto Delgado, Y., Herrera, N., Gallón, C., Álvarez, M. y Muñoz, L. \\ La responsabilité sociale des entreprises, un regard à sa mise en oeuvre dans le secteur public transport automobile}

Résumé: la Responsabilité Sociale d'Entreprise, la RSE - est l'un des concepts modernes qui a acquis plus d'importance au cours des dernières années dans le monde entier, en tant implique un engagement des organisations et entreprises pour le bien-être commun, cet article part de déterminer des pratiques de RSE qui applique le secteur public de transport dans la Vallée de Aburrá, en accord avec la GRI. Par conséquent, a identifié toutes les entreprises de transport consacrées à cette activité et a évalué les pratiques de RSE qui appliquent, en accord avec le guide.

Mots-clés: responsabilité sociale des entreprises, chaîne d'approvisionnement, l'environnement, logistique.

\section{A Responsabilidade Social Corporativa, um olhar para a sua aplicação no sector dos transportes públicos Automotivo}

Resumo: Responsabilidade Social Corporativa (CSR) é um dos conceitos modernos que adquiriu maior importância nos últimos anos em todo o mundo, em ambos implica um compromisso das organizações e empresas para o bem comum. Este artigo, dos resultados de investigação, parte identificando as práticas de RSE que aplica o sector de transportes públicos no vale de Aburrá automotivo, de acordo com as três dimensões do Guia Global Reporting Initiative - GRI: económico, social e ambiental, para o qual todas as empresas de transporte (ônibus) dedicados a esta actividade e implementação de práticas de RSE, de acordo com o guia identifica avaliada.

Palavras-chave: Responsabilidade Social Corporativa, transportes públicos, da Global Reporting Initiative (GRI), práticas de responsabilidade social empresarial e sustentabilidade. 


\section{Introducción}

La Responsabilidad Social Empresarial - RSE es un concepto desarrollado a lo largo del Siglo XX con amplios prospectos para el Siglo XXI, dado que las características del mercado han cambiado. En la actualidad para los consumidores son importantes aspectos trascendentes a los precios y a la calidad; y los impactos económicos, sociales y ambientales poco a poco se han convertido en aspectos diferenciadores al momento de adquirir productos y servicios.

Este artículo parte de identificar las prácticas de RSE que aplica el sector del transporte público automotor en el Valle de Aburrá, conforme con las tres dimensiones de la Guía Global Reporting Initiative - GRI: económico, social y ambiental; para tal fin se identificaron todas las empresas de buses dedicadas a esta actividad y se evaluaron las prácticas de responsabilidad social que aplican en concordancia con la guía.

Inicialmente se hace referencia teórica de la importancia de la RSE y del por qué la Guía GRI constituye el referente más acertado para revelar las actividades sociales, económicas y ambientales adelantadas por las empresas. Posteriormente, se presenta el contexto de las problemáticas del sector transporte público automotor en el Valle de Aburrá. Luego, se exponen la metodología empleada para la evaluación de las empresas, y los impactos generados en la sociedad por la implementación de prácticas responsables. Finalmente, se exponen las conclusiones encontradas a lo largo de este proceso de investigación.

\section{Metodología}

Para abordar el proceso de investigación se optó por un enfoque mixto, es decir, se combinaron métodos cualitativos y cuantitativos. El abordaje cualitativo se planteó de tipo descriptivo, mientras que lo cuantitativo se centró en el caso particular del sector automotor y las variables asociadas a sus características. Para el trabajo de campo se tomaron como muestra 53 empresas de transporte público automotor, que componen el total de empresas de buses dedicadas al transporte intermunicipal del Área Metropolitana del Valle de Aburrá, en Antioquia - Colombia.

Con el fin de desarrollar la etapa cualitativa se formuló una entrevista dirigida a los gerentes o directores de recursos humanos de las empresas de transporte público automotor, según la accesibilidad de la empresa y la disponibilidad 


\section{Contexto}

Delgado, Y., Herrera, N., Gallón, C., Álvarez, M. y Muñoz, L.

para brindar la información, con el fin de obtener un valor agregado a la investigación, dado que se consideró que con la aplicación de estas entrevistas se obtendría una mirada más profunda del sector. Se indagó por la opinión acerca de la RSE y, si en las empresas se aplicaban prácticas socialmente responsables. También se preguntó acerca de los posibles beneficios obtenidos con la aplicación de estas prácticas. Se buscó un acercamiento al modelo de presentación que actualmente utilizan las empresas para publicar las memorias de RSE.

En el tema cuantitativo se tomaron las 53 empresas como muestra, con el fin de establecer si brindaban algún tipo de información acerca de la aplicación de prácticas de RSE. Para ello se realizaron tres filtros: el primero, la búsqueda en las páginas web de información relacionada con la RSE; el segundo, buscar en las redes sociales (Facebook, Twitter); y el tercero, el contacto telefónico. El análisis de información tanto cualitativa como cuantitativa se realizó con el fin de observar si las empresas aplicaban prácticas sociales responsables y en caso de que lo hicieran, si los reportes generados a partir de las prácticas se encontraban realizados bajo el marco normativo de la Guía GRI con eje en los aspectos económico, social y ambiental, los cuales se describen a continuación:

\section{Guía GRI}

En el mundo existen diversos modelos de aplicación y manifestación de la RSE. Los mismos se han desarrollado e incursionado en las décadas comprendidas entre 1990 y 2010. Los modelos han surgido como respuesta a la necesidad de estandarizar y generar metodologías de aplicación.

En la Conferencia de las Naciones Unidas sobre el Medio Ambiente y el Desarrollo de 1992, en la que participaron 172 países y conocida como "La Cumbre de Río", se presentó una materialización importante en una serie de documentos que promueven la RSE. Los documentos generados se limitan al tema de la protección y conservación del ambiente. Entre ellos se pueden mencionar la Agenda 21 que promueve acciones para el cuidado de la naturaleza y el medio ambiente, y la Declaración de Principios Forestales que se encuentra bajo la misma línea. La Cumbre de Río dejó por fuera los principios económicos y sociales, pilares de la RSE actual.

En el año de 1998 es presentada la Social Accountability (SA) 8000, creada por una organización de los Estados Unidos llamada Responsabilidad Social Internacional - (SAI), su enfoque se encuentra en la promoción de prácticas responsables a través de toda la cadena de suministros, centradas en las 
condiciones laborales. Esta norma es de aplicación principal en los sectores agroindustriales, dejando por fuera los demás sectores y temas como el medio ambiente y el factor económico.

En el año de 1999 se presentan varias normas como:

- OHSAS 18001 con enfoque en los riesgos laborales y las condiciones de los empleados.

- AccountAbility 1000 (AA1000) norma para auditar los informes de RSE.

- AccountAbility 1000 con enfoque central en los stakeholders.

Las anteriores son normas que si bien abarcan parte de la RSE no responden al enfoque global desarrollado por el Global Reporting Initiative bajo las tres dimensiones que contempla la RSE: económica, social y ambiental.

El Pacto Global de las Naciones Unidas de 1999 es otro de los lineamientos que, en materia de RSE, se han estudiado y aplicado a nivel mundial. El Pacto se centra principalmente en el área de derechos humanos, los trabajadores, el ambiente y la lucha contra la corrupción; y no interviene el tema económico, sin tener en cuenta que el fin último de las empresas es generar riqueza, la misma que para la Guía GRI requiere de equilibrio en los tres aspectos mencionados. En el año 2000 surge la Guidelines for Multinational Enterprises dirigida a empresas multinacionales o con gran capacidad de operación. Si bien abarca en gran medida los tres aspectos para lograr el equilibrio y el desarrollo sostenible, sectoriza la aplicación a grandes empresas, dejando de lado las de menor tamaño, que si bien no tienen las mismas capacidades financieras que las multinacionales, componen mucho más de las tres cuartas partes de las empresas a nivel mundial y son parte integral de la economía.

Por su parte, la Guía GRI se fundamenta en tres pilares fundamentales: el desempeño ambiental, económico y social. De esta manera fusiona los temas fundamentales para las empresas y los temas que interesan a los diversos grupos de interés impactados por las acciones llevadas a cabo. Para dar cumplimiento cabal en todos los aspectos de la Guía es necesario revelar información muy precisa acerca de los desempeños y acciones de los tres aspectos. La Guía propone un conjunto de categorías, subcategorías y aspectos, medidos a partir de indicadores enmarcados bajo los tres principios que buscan el equilibrio. De esta manera, se garantiza a la sociedad la aplicación de prácticas que propendan por el equilibrio económico, social y ambiental, y que no sólo beneficien los intereses monetarios de las compañías sino que además garanticen el desarrollo sostenible de las comunidades. 


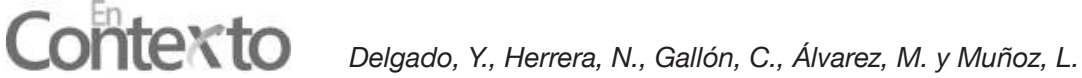

Además de lo anterior, la Guía GRI proporciona elementos que permiten evaluar la eficiencia operativa de la empresa en términos de RSE, evaluando indicadores como el consumo de recursos, lo cual pone de manifiesto la necesidad de ahorrar en busca de reducir gastos que permitan no solo aumentar los rendimientos sino además disminuir el impacto generado al ambiente. La Guía GRI realiza revelaciones de tipo económico que bajo las actuales Normas Internacionales de Información Financiera (NIIF) son importantes. Estas normas obligan a revelar una serie de hechos acerca del desempeño económico de las compañías, permitiendo a los lectores y usuarios de interés obtener mayor información y claridad con respecto a los temas financieros.

\section{Resultados}

Los crecientes efectos del desarrollo de las economías en todos los países han provocado efectos negativos en el medio ambiente y en la sociedad. Esto hace pensar que el crecimiento económico sin ningún control y sin responsabilidades éticas acarrea consecuencias desastrosas para los seres humanos. La rápida industrialización y el consumo acelerado de recursos de todo tipo han llevado al planeta hacia una carrera insostenible ambientalmente. El consumo se convirtió en el nuevo dios de las personas y el bienestar social es asociado a la cantidad de bienes o productos consumidos. El poder se ha traducido en tener, y el tener en malgastar los bienes, consumiendo desproporcionadamente sin dimensionar las nefastas consecuencias que ello acarrea. Los continuos cambios de moda y el acelerado avance tecnológico han llevado a la sociedad al consumo masivo de recursos; éstos son utilizados para producir y seguidamente aumentar la fabricación, acrecentando el consumo de energía, generando más gases de efecto invernadero. Toda una cadena insostenible desde cualquier óptica.

Garantizar el bienestar de las generaciones futuras es un deber de los seres humanos que hoy habitan la tierra. Lo anterior es definido en la actualidad como desarrollo sostenible: "capacidad de un individuo, organización o sociedad para satisfacer las necesidades de las generaciones presentes, sin comprometer las posibilidades de las del futuro para atender sus propias necesidades" (Brundtland, 1897). Este concepto invita a pensar en las acciones realizadas en el presente, más allá de intereses individualistas que priven las condiciones y calidad de vida de las generaciones futuras.

El desarrollo sostenible hace parte fundamental de la responsabilidad social y más concretamente de la RSE, ya que en gran medida las políticas empresariales y el afán por generar utilidades a cualquier costo, poco a poco han puesto sobre la mesa las consecuencias que dichas acciones acarrean. De esta manera, la 
RSE ha surgido como noción en los tiempos recientes a partir de una serie de construcciones conceptuales de las acciones que deben tomar las compañías para aportar al desarrollo económico sostenible, entendiéndose éste como el pilar para construir un futuro en equilibrio económico, social y ambiental.

No es posible hablar de desarrollo sostenible sin unirlo con la RSE, entendiéndose ésta como:

Un conjunto de prácticas que asumen las empresas con la finalidad de armonizar y lograr un equilibrio entre las dimensiones de rentabilidad económica, derechos humanos, bienestar social y protección ambiental. De esta manera se pretende que las empresas contribuyan, conjuntamente con el estado y la sociedad civil, a la construcción de una sociedad más justa, equitativa y sustentable (Baltera y Díaz, 2005, p.22).

Sin embargo, la RSE no es una filosofía empresarial altruista y filantrópica como tal, sino que conjuga elementos de las características que se mencionan y tiene como fin último la sostenibilidad y rentabilidad empresarial. Por ello, la RSE es desarrollada desde la planeación estratégica, con principios esbozados por la alta administración de las compañías.

\section{Filantropía y RSE}

Dada la naturaleza de los beneficios fiscales al aplicar prácticas de RSE, se puede pensar que la RSE no existe por sí misma sino por las retribuciones que en materia económica pueden ser obtenidas. En este sentido, se hace necesario distinguir entre los conceptos de RSE y filantropía. En el primer caso, se trata del desarrollo de actividades que en materia económica, social y ambiental contribuyen al mejoramiento de las condiciones de vida de las diversas partes involucradas (empleados, proveedores, consumidores, inversionistas, entre otros) llamados también stakeholders, favoreciendo el equilibrio ambiental y el desarrollo sostenible, pretendiendo obtener beneficios económicos futuros derivados de dichas actividades. Además, la RSE parte de la planeación estratégica, con presupuesto definido y buscando retribuciones económicas hacia el futuro, con políticas claras dentro de las organizaciones que permitan su aplicación, mantenimiento y desarrollo en el tiempo.

Por su parte, la filantropía hace referencia a acciones puntuales sin planeación y sin concordancia con los objetivos estratégicos de las organizaciones, de las cuales no se espera ningún tipo de retribución económica. De esta manera, el objetivo primordial de las empresas no se desvirtúa por la implementación de 
prácticas sociales responsables, ya que indiscutiblemente se espera retribución económica, con la diferencia de estar contribuyendo al desarrollo económico sostenible.

Tabla 1

Diferencias entre la RSE y la filantropia

\section{RESPONSABILIDAD SOCIAL EMPRESARIAL}

Es una política de empresa.

\section{FILANTROPÍA}

Es una política de personas: el propietario, los accionistas.
Es una acción permanente. Es un modo de actuar y de hacer empresa.
Es una acción de carácter puntual.

\footnotetext{
Es un compromiso formal de carácter voluntario, Es una acción voluntaria no sujeta a evaluación sujeto a evaluación y control social. ni control social.
}

Tiene una dimensión externa e interna.

Tiene una dimensión solo externa.

\begin{tabular}{|c|c|}
\hline $\begin{array}{l}\text { Se basa en principios éticos. El reconocimiento de } \\
\text { las personas y sus derechos. }\end{array}$ & $\begin{array}{l}\text { Se basa en principio altruistas, como } \\
\text { la solidaridad. }\end{array}$ \\
\hline $\begin{array}{l}\text { Se orienta a personas (sus trabajadores, } \\
\text { proveedores, clientes), a la comunidad y a la } \\
\text { sociedad en general. }\end{array}$ & $\begin{array}{l}\text { Se orienta a instituciones o a la sociedad } \\
\text { civil organizada: fundaciones, universidades, } \\
\text { organizaciones de ayuda solidaria. }\end{array}$ \\
\hline $\begin{array}{l}\text { Tiene y se busca un impacto económco en la } \\
\text { empresa: mayor producitividad, mayores ventas, } \\
\text { mayores ganancias. }\end{array}$ & $\begin{array}{l}\text { No tiene ni se pretende un impacto económico } \\
\text { en la empresa }\end{array}$ \\
\hline $\begin{array}{l}\text { Apunta a generar una relación virtuosa entre las } \\
\text { empresas y entre la empresa, la comunidad, el } \\
\text { gobierno, la sociedad y el medio ambiente. }\end{array}$ & $\begin{array}{c}\text { No busca generar una relación virtuosa en la } \\
\text { cadena productiva, ni con la sociedad } \\
\text { y sus agentes. }\end{array}$ \\
\hline $\begin{array}{l}\text { Los recursos financieros destinados al } \\
\text { financiamiento de las acciones de responsabilidad } \\
\text { social, provienen del presupuesto de la empresa. }\end{array}$ & $\begin{array}{l}\text { Los recursos financieros que se destinan a las } \\
\text { acciones filantrópicas, provienen de las utilidades. }\end{array}$ \\
\hline
\end{tabular}

Baltera y Díaz, 2005.

\section{La RSE, el marketing social y la economía solidaria}

La RSE también se confunde con conceptos como el marketing social y la economía solidaria. Con respecto al primero, es necesario hacer la diferenciación, ya que a simple vista la palabra puede inducir a crear una idea de tratarse de un mercadeo responsable en favor de los grupos de interés, significado enfocado hacia las prácticas de RSE; si bien en algo se asemeja no es propiamente lo que plantea. 
El marketing social puede considerarse como el diseño, implementación y control de programas ideados para influir en la aceptabilidad de ideas sociales, implicando consideraciones de planeación de productos, precios, comunicación, distribución e investigación de mercados (Kloter y Zaltman, 1971). Dicho marketing ha sido utilizado con el fin de influir en los comportamientos y decisiones de las personas, comprometiendo el comportamiento a la obtención de beneficios personales y sociales. Lo que se pretende es que las personas se vean favorecidas a partir de un cambio en la manera de actuar. El marketing social no se enfoca en la idea de vender un producto o servicio, se orienta hacia los beneficios que se pueden obtener con un cambio en el modo de comportarse.

Si bien se utilizan técnicas del mercadeo comercial (como la identificación del segmento de mercado, caracterización de los individuos, análisis de los obstáculos que se pueden generar en la implementación u ofrecimiento del producto o servicio y la publicidad como medio para dar a conocer el producto o servicio), en el marketing social no se busca cambiar comportamientos propios para lucrar intereses comerciales. En ese orden de ideas, el lucro de forma monetaria queda desvirtuado como aplicación conceptual del marketing social.

Las prácticas de RSE proponen un mercadeo responsable, enfocado en la transparencia, el cumplimiento regulatorio y la debida información en la comercialización de bienes y servicios, conservando como objetivo el beneficio económico y sostenibilidad de las empresas en el largo plazo.

Por su parte, la economía solidaria surge de los problemas originados por el sistema capitalista, buscando brindar alternativas frentea la concentración de la riqueza en unas pocas personas y reconociendo un "sistema socioeconómico, cultural y ambiental, conformado por el conjunto de fuerzas sociales organizadas en formas asociativas, identificadas por prácticas autogestionarias solidarias, democráticas y humanistas, sin ánimo de lucro, para el desarrollo integral del ser humano como sujeto, actor y fin de la economía" (Artículo 2, Ley 454, 1998).

\section{El transporte, un medio para el desarrollo y la "piedra en el camino" de las grandes urbes}

El sector transporte comprende, en Colombia, actividades terciarias o de prestación de servicios, la cual incluye el transporte de carga, marítimo, aéreo, terrestre y por supuesto el servicio de transporte público. Esta actividad económica es uno de los principales factores que determinan la competitividad de un país, ya que su desarrollo permite la comunicación y el desplazamiento 


\section{Contexto}

Delgado, Y., Herrera, N., Gallón, C., Álvarez, M. y Muñoz, L.

hacia las diferentes zonas o regiones del territorio, haciendo posible y facilitando las actividades de comercio, turismo, construcción, entre otras.

Así como este sector contribuye en gran manera al desarrollo económico y social, también aporta a la contaminación del medio ambiente, y por esto debe no solo apoyar con el factor estructural y económico sino también con el factor medio ambiental. Una forma de hacerlo es proveer prácticas sociales responsables en las empresas de transporte público automotor, que impliquen pasar del hecho individual de transportarse al hecho de reconocer los impactos económicos, sociales y ambientales que implican el desarrollo de la actividad.

El sector transporte ha mostrado cumplir sus metas en un $92 \%$, según el Ministerio de Transporte (2014), quien indicó que Invías pavimentó 1.703 $\mathrm{km}$, construyó $33 \mathrm{~km}$ de dobles calzadas, 117 puentes y 15,5 km de túneles; y realizó el mantenimiento y rehabilitación vial de $2.241 \mathrm{~km}$ de vías. Para el año 2014, se esperaba alcanzar la pavimentación de 2.192 km, la construcción de 62 $\mathrm{km}$ de dobles calzadas, 183 puentes y $18,6 \mathrm{~km}$ de túneles, y el mantenimiento y rehabilitación vial de $2.745 \mathrm{~km}$. Además la entidad aumentó sus actividades de vigilancia e incrementó sus visitas de inspección en un 271\% y 588\% respectivamente, en las que se incluyen por primera vez operativos encubiertos en transporte intermunicipal y operativo en vías nacionales con Policía de Tránsito y Transporte.

Los problemas de movilidad del Valle de Aburrá van más allá del tema de construcción de vías para el uso de los vehículos. La accidentalidad, la disminución de la competitividad, el aumento en los tiempos de desplazamiento, aumento del consumo energético, la disminución de la cantidad de metros cuadrados públicos para el disfrute dado el aumento de vías, el deterioro de la malla vial y la congestión, combinados con la incultura ciudadana, hacen de la movilidad una bomba de tiempo que no parece detenerse.

En este sentido, es importante hacer un análisis cualitativo en el cual se puedan triangular las opiniones que se encontraron y que se pueden resumir en tres direcciones: las empresas que plantean que la RSE es un engaño de la era contemporánea y una irresponsabilidad por parte de las empresas que lo practican; están las empresas que consideran que es una práctica que se asume buscando equilibrio entre finalidad económica y un desarrollo sostenible; y por último, están las empresas que su razón de ser es cooperativa, por lo cual, su concepción misma es social, económica y solidaria.

A pesar que se reconoce la importancia en el mejoramiento de la imagen corporativa y en la sostenibilidad de la empresa en el largo plazo, se conserva 
la concepción de que aplicar prácticas de RSE es un gasto para la compañía y no una inversión. Igualmente, el factor salario y la retribución justa juegan un papel fundamental en la concepción de aplicación de prácticas socialmente responsables. Para las empresas, las prácticas socialmente responsables empiezan por allí. Desconocer que la retribución justa a los empleados incide de manera directa en los índices de productividad y ambiente laboral, implicaría dejar de lado la categoría de desempeño social propuesta por la Guía GRI.

De esta manera, se logró establecer que si bien las empresas no tienen un profundo conocimiento del concepto de RSE, de manera empírica realizan la aplicación de prácticas socialmente responsables en búsqueda de diversos objetivos, teniendo presente a la comunidad y el medio ambiente como foco de atención.

Actualmente en el Valle de Aburrá prestan el servicio de transporte público automotor 53 empresas de buses que cubren las rutas a lo largo y ancho de toda el Área Metropolitana, ${ }^{1}$ las cuales tienen conocimiento de las problemáticas y los impactos del servicio que prestan, y lo que ocasiona en materia ambiental, social y económica; pero sólo 13 empresas realizan prácticas socialmente responsables, lo cual equivale al $25 \%$ de las empresas del sector, como muestra la Figura 1.

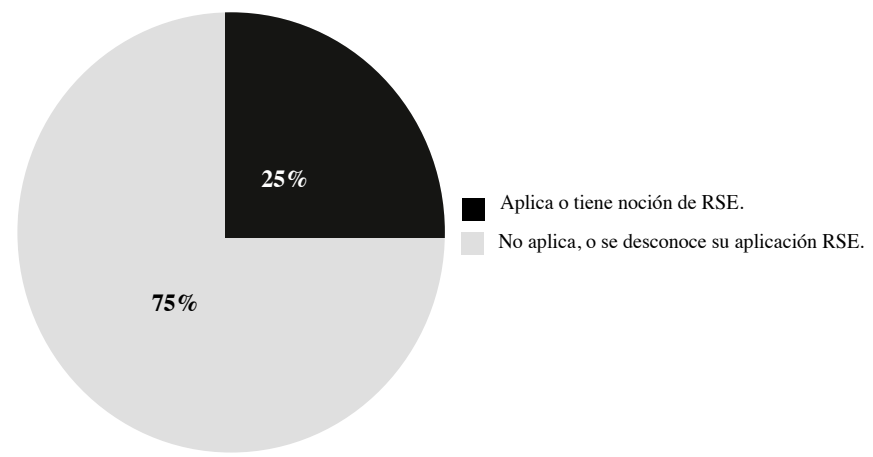

Figura 1. Empresas de transporte público que aplican RSE

Elaboración propia.

1 La conforman los municipios de Barbosa, Copacabana, Bello, Medellín, Envigado, Sabaneta, Itagüí, La Estrella y Caldas. 


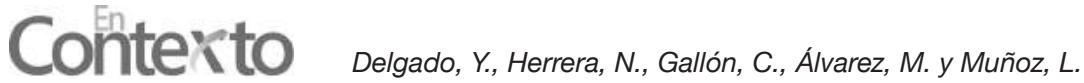

Con referencia al rastreo de información por medio de las páginas web de las empresas consultadas acerca de las prácticas de RSE, se encontró que el 17\% de las empresas de transporte público automotor en el Valle de Aburrá publican en sus páginas información relacionada con prácticas o actividades de RSE. Para detallar aún más esta información se muestra la Figura 2.

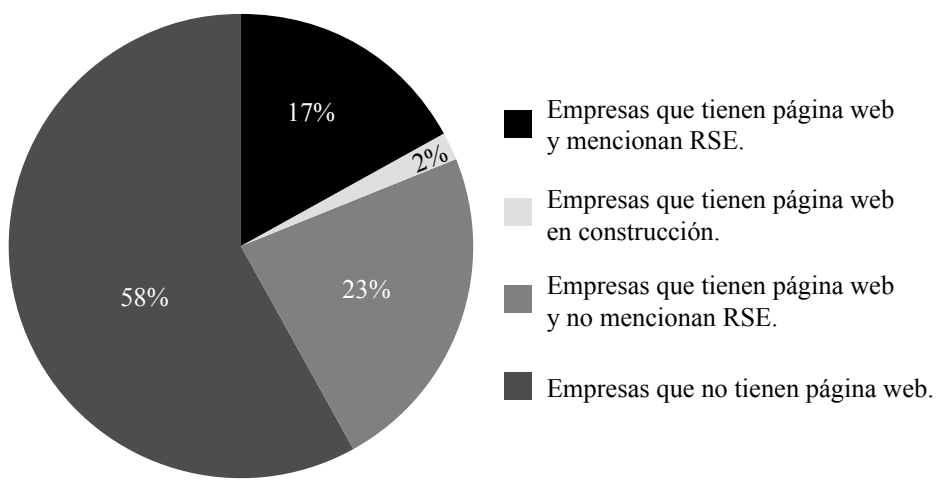

Figura 2. Empresas de transporte público que divulgan RSE en sus páginas web Elaboración propia.

Respecto de la búsqueda en las redes sociales (Facebook, Twitter) de las 53 empresas de transporte automotor del Valle de Aburrá, se encontró que 18 tienen Facebook, pero allí no publican información sobre RSE. En cuanto al contacto telefónico, de las 53 empresas de trasporte automotor del Valle de Aburráse logró establecer comunicación con 38.

Tabla 2

Contacto telefónico con empresas de transporte público del Valle de Aburrá

\begin{tabular}{ll}
\hline Resultado & Número de empresas \\
\hline $\begin{array}{l}\text { Dieron su correo electrónico para solicitar la información } \\
\text { a través de este medio (se obtuvieron 5 respuestas). }\end{array}$ & 14 \\
No se puede brindar la información. & 3 \\
No se pudo lograr la comunicación. & 5 \\
Desconocen el tema. & 7 \\
Brindaron información en la llamada. & 5 \\
No presentan este tipo de información. & 4 \\
\hline
\end{tabular}

Elaboración propia. 
Gracias al rastreo de información en páginas web, al contacto telefónico y a las entrevistas realizadas, se encontraron unos listados de actividades que fueron clasificados de acuerdo con el GRI, ya que se ajustaban a las categorías, sub categorías, aspectos e indicadores del Global Reporting Initiative. Si bien no se pudo tener acceso a informes de gestión ni a informes sociales, con la información obtenida en las páginas web, en las llamadas y en las entrevistas se pudo construir un cuadro relacional donde se muestran cuáles son los aspectos del GRI que pone en práctica el sector transporte automotor del Valle de Aburrá.

\begin{tabular}{|c|c|c|c|c|c|}
\hline \multicolumn{6}{|c|}{ Categorías y aspectos de la Guía aplicados por las empresas del sector transporte automor del Valle de Aburrá } \\
\hline Categoría & \multicolumn{3}{|l|}{ Enonomía } & \multicolumn{2}{|c|}{ Medio Ambiente } \\
\hline Aspectos & \multicolumn{3}{|c|}{$\begin{array}{l}\text { - Economía } \\
\text { - Desempeño económico } \\
\text { - Presencia en el mercado } \\
\text { - Consecuencias económicas } \\
\text { indirectas }\end{array}$} & \multicolumn{2}{|c|}{$\begin{array}{l}\text { - Energía } \\
\text { - Emisiones } \\
\text { - Transporte } \\
\text { - Biodiversidad } \\
\text { - Productos y servicios } \\
\text { - Mecanismos de reclamación en materia } \\
\text { ambiental } \\
\text { - Efluentes y residuos }\end{array}$} \\
\hline \multicolumn{6}{|c|}{ Categoría: desempeño social } \\
\hline Subcategorías: & $\begin{array}{l}\text { Prácticas laborales y } \\
\text { trabajo digno }\end{array}$ & Dere & los humanos & Sociedad & $\begin{array}{l}\text { Responsabilidad } \\
\text { sobre productos }\end{array}$ \\
\hline Aspectos & $\begin{array}{l}\text { - Capacitación y } \\
\text { Educación } \\
\text { - Empleo } \\
\text { - Mecanismos de } \\
\text { reclamación sobre las } \\
\text { prácticas laborales }\end{array}$ & $\begin{array}{l}\text { - Mec } \\
\text { reclan } \\
\text { en ma } \\
\text { derech }\end{array}$ & $\begin{array}{l}\text { ismos de } \\
\text { ción } \\
\text { ria de } \\
\text { sumanos }\end{array}$ & $\begin{array}{l}\text { - Comunidades locales } \\
\text { - Mecanismos de } \\
\text { reclamación por } \\
\text { impacto social }\end{array}$ & $\begin{array}{l}\text { - Salud y seguridad } \\
\text { de los clientes }\end{array}$ \\
\hline
\end{tabular}

Figura 3. Aspectos del GRI que pone en práctica el Sector Transporte Automotor del Valle de Aburrá

Elaboración propia.

La Guía GRI se fundamenta en tres pilares: el desempeño ambiental, el económico y el social; buscando un equilibrio entre el impacto económico sin descuidar los aspectos sociales y ambientales, construyendo una trilogía armoniosa en pro de la organización y sus stakeholders. El sector transporte automotor aborda de una u otra manera las tres categorías del GRI, siendo el desempeño social la categoría a la que más le apunta el sector, lo cual se evidencia en el listado de actividades de RSE identificadas en este trabajo 


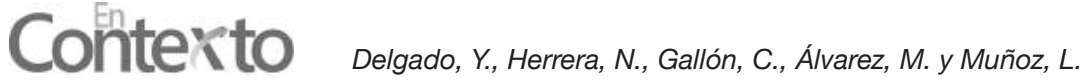

de investigación, en el cual los indicadores más frecuentes y que mejor se acoplan a las actividades realizadas por el Sector Transporte Automotor del Valle de Aburrá son los de la categoría desempeño social; sin olvidar que toda actividad que se realice en términos de RSE tiene un costo económico en la organización, por lo cual afecta directamente la categoría de desempeño económico, más puntualmente el indicador G4-EC1: Valor económico directo generado y distribuido.

De las 56 actividades identificadas de RSE que realiza el Sector Automotor del Valle de Aburrá, 26 se ejecutan en la categoría desempeño social, 8 combinan el tema económico y social, 7 son netamente económicas, 8 de la categoría de medio ambiente, 1 combina lo económico y lo ambiental, 5 corresponden a acciones filantrópicas y 1 aplica las tres categorías (económica, social y ambiental). Esta última acción corresponde a la aplicación del sistema de gestión de calidad con certificación ISO 9001:2008.

En las actividades encontradas en el rastreo de información algunas de ellas no se ajustaban al concepto de RSE y estaban más relacionadas con la filantropía, estas actividades son las siguientes:

- Actividades destinadas a promover el ejercicio espiritual y de fe.

- Entrega de mercados (abarrotes).

- Prestación del servicio gratuito para funerales y capacitaciones.

- Espacio para actividades de sano esparcimiento de los empleados y su grupo familiar.

- Ayuda a las Juntas de Acción Comunal.

Del análisis de la información también se pudo identificar una serie de actividades, como se mencionó anteriormente, que se pueden relacionar con algunos indicadores de la Guía GRI, los cuales presentan a continuación. 
Tabla 3

Actividades relacionadas con indicadores de la Guía GRI

Actividades

Indicadores del GRI

Pago justo y adecuado a los trabajadores.
G4-EC5: Relación entre el salario inicial desglosado por sexo y el salario mínimo local en lugares donde se desarrollan operaciones significativas.

G4-EC1: Valor económico directo generado y distribuido.

G4-EC3: Cobertura de las obligaciones de la organización derivadas de su plan de prestaciones.

G4-LA2: Prestaciones sociales para los empleados a jornada completa que no se ofrecen a los empleados temporales o a media jornada, desglosado por ubicaciones significativas de actividad.

Incrementar la seguridad vial y disminuir los índices de accidentalidad.
G4-PR1: Porcentaje de categorías de productos y servicios significativos cuyos impactos en materia de salud y seguridad se han evaluado para promover mejoras.

G4-SO1: Porcentaje de operaciones donde se han implantado programas de desarrollo, evaluaciones de impactos y participación de la comunidad local.
Trabajar en conjunto con las entidades públicas de transporte buscando mejorar las necesidades de los usuarios.
G4-SO1: Porcentaje de operaciones donde se han implantado programas de desarrollo, evaluaciones de impactos y participación de la comunidad local.

G4-SO2: Centros de operaciones con efectos negativos significativos, posibles o reales, sobre las comunidades locales.
Subsidios otorgados por la empresa (vivienda, educación, adquisición de equipos tecnológicos).
G4-EC1: Valor económico directo generado y distribuido. G4-SO1: Porcentaje de operaciones donde se han implantado programas de desarrollo, evaluaciones de impactos y participación de la comunidad local.
Fondos para el pago de auxilio por quema, hurto, volcamiento y terrorismo.
G4-EC1: Valor económico directo generado y distribuido.

G4-SO1: Porcentaje de operaciones donde se han implantado programas de desarrollo, evaluaciones de impactos y participación de la comunidad local. 
Actividades

Vinculación con las

Comunidades, apoyo al adulto mayor, grupos juveniles, convites

barriales y comunidades

religiosas.

\section{Indicadores del GRI}

Control en la emisión de gases.
G4-SO1: Porcentaje de operaciones donde se han implantado programas de desarrollo, evaluaciones de impactos y participación de la comunidad local.

G4-EN15: Emisiones directas de gases de efecto invernadero (alcance 1).

G4-EN19: Reducción de las Emisiones de Gases de Efecto Invernadero.

G4-EN3: Consumo Energético Interno.

G4-EN6: Reducción del consumo energético.

Construcción de vías. G4-EC7: Desarrollo e impacto de la inversión en infraestructuras y los tipos de servicios.

G4-S01: Porcentaje de operaciones donde se han implementado programas de desarrollo, evaluación de impactos y participación de la comunidad local.

Movilidad de productos, movilidad de trabajadores y estudiantes.

Actividades de promoción y prevención en salud para los empleados, sus familias y la comunidad más necesitada.
G4-EN30: Impactos ambientales significativos del transporte de productos y otros bienes y materiales utilizados para las actividades de la organización, así como del transporte de personal.

G4-LA5: Porcentaje de trabajadores que está representado en comités formales de seguridad y salud conjuntos para dirección y empleados, establecidos para ayudar a controlar $\mathrm{y}$ asesorar sobre programas de seguridad y salud laboral.

G4-LA7: Trabajadores cuya profesión tiene una incidencia o un riesgo elevados de enfermedad.

Capacitación teórico práctica en técnicas de conducción.
G4-LA9: Promedio de horas de capacitación anuales por empleado, desglosado por sexo y por categoría laboral.

G4-LA10: Programas de gestión de habilidades y de formación continuada que fomentan la empleabilidad de los trabajadores y les ayudan a gestionar el final de sus carreras profesionales. 


\section{Actividades}

\section{Indicadores del GRI}

Actividades para fomentar estilos de vida saludables a través de deporte y actividades recreativas para los empleados y su grupo familiar.
G4-LA5: Porcentaje de trabajadores que está representado en comités formales de seguridad y salud conjuntos para dirección y empleados, establecidos para ayudar a controlar y asesorar sobre programas de seguridad y salud laboral.

G4-LA7: Trabajadores cuya profesión tiene una incidencia o un riesgo elevados de enfermedad.
Centro de recolección de residuos; destinado para garantizar el manejo adecuado de los residuos generados en la empresa y su almacenamiento.
G4-EN23: Peso total de residuos gestionados, según tipo y método de tratamiento.

Tiquete estudiantil.

G4-S01: Porcentaje de operaciones donde se han implementado programas de desarrollo, evaluación de impactos y participación de la comunidad local

Dotación de uniformes para los equipos de fútbol del sector.
G4-S01: Porcentaje de operaciones donde se han implementado programas de desarrollo, evaluación de impactos y participación de la comunidad local.

Arreglos en la vía.

G4-S01: Porcentaje de operaciones donde se han implementado programas de desarrollo, evaluación de impactos y participación de la comunidad local.

G4-EC7: Desarrollo e impacto de la inversión en infraestructuras y los tipos de servicios.

Caminata ecológica. G4-EN13: Hábitats protegidos o restaurados. G4-S01: Porcentaje de operaciones donde se han implementado programas de desarrollo, evaluación de impactos y participación de la comunidad local.

Fiesta de integración (novenas navideñas, fiestas de fin de año, día del conductor).
G4-EC1: Valor económico directo generado y distribuido. G4-SO1: Porcentaje de operaciones donde se han implantado programas de desarrollo, evaluaciones de impactos y participación de la comunidad local. 
Actividades

Solo se contrata personas de la comunidad.

\section{Indicadores del GRI}

Actividades deportivas.
G4-SO1: Porcentaje de altos directivos procedentes de la comunidad local en lugares donde se desarrollan operaciones significativas.

G4-LA5: Porcentaje de trabajadores que está representado en comités formales de seguridad y salud conjuntos para dirección y empleados, establecidos para ayudar a controlar y asesorar sobre programas de seguridad y salud laboral.

G4-LA7: Trabajadores cuya profesión tiene una incidencia o un riesgo elevados de enfermedad.

Servicio de transporte gratuito para los grupos de tercera edad, instituciones educativas y comunidades de bajos recursos, asilos, parroquias y policía comunitaria.
G4-SO1: Porcentaje de centros donde se han implantado programas de desarrollo, evaluaciones de impactos y participación de la comunidad local.
Protección del medio ambiente mediante la implementación de vehículos a gas natural.
G4-EN6: Reducción del consumo energético.

G4-EN7: Reducciones de los requisitos energéticos de los productos y servicios.

G4-EN19: Reducción de las emisiones de gases efecto invernadero.
Jornadas de salud para el empleado y su grupo familiar, con actividades de prevención, promoción y mejoramiento de las condiciones físicas de cada uno de sus integrantes.
G4-LA5: Porcentaje de trabajadores que está representado en comités formales de seguridad y salud conjuntos para dirección y empleados, establecidos para ayudar a controlar y asesorar sobre programas de seguridad y salud laboral.

G4-LA7: Trabajadores cuya profesión tiene una incidencia o un riesgo elevados de enfermedad. 
Actividades

Donaciones a comunidades de escasos recursos.

\section{Indicadores del GRI}

G4-EC1: Valor económico directo generado y distribuido (a pesar que se le puede aplicar este indicador de la Guía.

GRI: la donación es una actividad muy relacionada a la filantropía, debe estar muy estructurado en la planeación estratégica de la empresa para que se considere RSE).
Acompañamiento psicológico.
Salud y seguridad en el trabajo.*

Mecanismos de reclamación por impacto social.*

Página web de preguntas, quejas y reclamos de los clientes.

Plantación de árboles. G4-EN12: descripción de los impactos más significativos en la biodiversidad de áreas protegidas o áreas de alto valor en términos de diversidad biológica no protegidas derivados de las actividades, productos y los servicios.

G4-EN13: Hábitats protegidos o restaurados.

Renovación urbana. G4-EC7: Desarrollo e impacto de la inversión en infraestructuras y los tipos de servicios.

G4-EC8: Impactos económicos indirectos significativos y alcance de los mismos.

Sistema de PQRS.

G4-EN34: Número de reclamaciones ambientales que se han presentado, abordado y resuelto mediante mecanismos formales de reclamación.

G4-LA16: Número de reclamaciones sobre prácticas laborales que se han presentado, abordado y resuelto mediante mecanismos formales de reclamación.

G4-HR12: Número de reclamaciones sobre derechos humanos que se han presentado, abordado y resuelto mediante mecanismos formales de reclamación.

G4-SO11: Número de reclamaciones sobre impactos sociales que se han presentado, abordado y resuelto mediante mecanismos formales de reclamación. 
Actividades

\section{Indicadores del GRI}

Apoyo a actividades culturales.
G4-EC1: Valor económico directo generado y distribuido (la actividad es algo ambigua se encuentra en los límites de la filantropía y la RSE).
Planes de manejo socio ambiental.
G4-EN27: Mitigación del impacto ambiental de los productos y servicios.

G4-EN34: Número de reclamaciones ambientales que se han presentado, abordado y resuelto mediante mecanismos formales de reclamación.

*No se clasifica en ningún indicador directo de GRI solo se clasifica en el aspecto.

Elaboración propia.

\section{La RSE, de la teoría a la práctica}

En Colombia existen varios casos de aplicación de prácticas sociales responsables, entre los que se destacan los desarrollados por las compañías petroleras como Ecopetrol y Pacific Rubiales, quienes dado el impacto ecológico que sus procesos involucran, buscan retribuir a la sociedad y disminuir los impactos ambientales. Ambas empresas, cuentan con programas definidos y con principios propuestos por la Guía GRI. Los resultados de ambas empresas en materia de desarrollo sostenible son para resaltar, dado el alto impacto ambiental que generan.

En México existe CEMEFI, una asociación civil sin fines de lucro, que se ha encargado desde el año 2000 de promover las prácticas sociales responsables aplicadas en América Latina, involucrando conceptos como la cadena de valor, la gestión social y ambiental. Anualmente se realiza premiación a las empresas y de acuerdo con las mismas, la RSE se ha convertido en una filosofía de vida, en la que se busca el desarrollo sostenible.

En España se encuentra el Observatorio de Responsabilidad Social Corporativa, como organismo sin ánimo de lucro, que propende por la aplicación de prácticas socialmente responsables, con el objetivo de lograr el equilibrio en los ámbitos social, económico y ambiental. 
Sin embargo, y a pesar de que la literatura busca conjugar los temas de RSE y desarrollo sostenible, no se logró hallar un estudio que relacione directamente las variables de ambos conceptos, cuantificando en materia financiera los beneficios a los cuales se verían abocadas las empresas al aplicar prácticas de RSE.

Respecto a los hallazgos, se observó que las prácticas de RSE implementadas principalmente en dos de las empresas analizadas, han sido sobresalientes y marcan el camino a seguir de las demás empresas de transporte en el Valle de Aburrá. No es un secreto que en el Área Metropolitana la concepción de modelo de transporte ha ido migrando hacia la integralidad de los sistemas. De esta manera, los organismos gubernamentales han exigido a las empresas de transporte la puesta en marcha de procesos que involucren a todos los sistemas de transporte. Los temas relacionados con prácticas de RSE como la conservación del medio ambiente, el uso correcto de los recursos, el cumplimiento de la normatividad vigente, la búsqueda de satisfacción de necesidades mínimas de los empleados, el respeto por las normas laborales, la contribución social en temas que sean de impacto directo a la comunidad, entre otras, son elementos que constituyen el eje central en la evaluación de los modelos a proponer en la integración del sistema de transporte en el Valle de Aburrá. Es hora de unir la rentabilidad y el desarrollo sostenible, el camino se encuentra dictado por las prácticas de RSE.

\section{Conclusiones}

La RSE en el sector transporte automotor es un fenómeno incipiente al que apenas algunas empresas empiezan a acercarse. Del estudio realizado surgen una serie de interrogantes: ¿Son las empresas de transporte automotor del Valle de Aburrá socialmente responsables? ¿Presentan informes de RSE? ¿Qué modelo o guía utilizan para presentar los informes? ¿No los publican porque consideran que contienen información confidencial? Estos interrogantes se quedan en el aire debido a la poca información y conocimiento que manifiestan las empresas sobre el tema.

De la información obtenida por medio del rastreo realizado en las páginas web, contacto telefónico y entrevistas, se pudo inferir que el tema de RSE es nuevo para el sector, al que apenas algunas empresas están empezando a incursionar por medio de actividades que buscan mantener un equilibrio entre obtener un impacto económico (mayor productividad, mayores ventas, mayor ganancia), un equilibrio ambiental y un desarrollo sostenible de su entorno.

Es de aclarar que de este estudio no puede surgir un estándar ni generalizaciones para el sector, debido a la poca información obtenida; 


\section{Contexto}

Delgado, Y., Herrera, N., Gallón, C., Álvarez, M. y Muñoz, L.

pero se puede resaltar que del $25 \%$ de las empresas de las que se obtuvo información, se evidencia una aplicación de la RSE de manera empírica, es decir, sin sustentos metodológicos, ni teóricos. No obstante, las actividades que realizan se ajustan a los lineamientos y planteamientos del GRI debido a que se incluyen los tres pilares fundamentales de la Guía GRI y, como se mostró en la investigación, se pueden ajustar a las categorías, subcategorías, aspectos e indicadores del informe.

\section{Referencias}

Alcaldía de Medellín. (2014). Medellín Cómo Vamos: Movilidad y espacio público. Recuperado de http://www.medellincomovamos.org/movilidady-espacio-p-blico.

Álvarez, M. y Medina, A. (2009). Responsabilidad Social Empresarial de grandes empresas manufactureras del área metropolitana del Valle de Aburrá. (Tesis de maestría). Universidad Pontificia Bolivariana, Medellín, Colombia.

Baltera, P. y Díaz, E. (2005). Responsabilidad social empresarial alcances y potencialidades en materia laboral. Cuaderno de investigación, 25, 1-164. Recuperado de http://www.dt.gob.cl/1601/articles-88984_recurso_1.pdf

Friedman, M. (1971). La responsabilidad social de la empresa privada. Recuperado de http://archipielagolibertad.org/upload/files2/002\%20 Propiedad $\% 20$ privada $/ 2.3 \% 20$ Responsabilidad $\% 20$ Social $\% 20$ Empresarial/0001\%20Friedman\%20-\%20La\%20esponsabilidad\%20 social $\% 20$ de $\% 201 \mathrm{a} \% 20$ empresa $\% 20$ privada.pdf

Fronti, I. (2012). Problemas de la Contabilidad Social y Ambiental: Algunas Reflexiones Sobre la Necesidad de un Abordaje Interdisciplinario. Contaduría Universidad de Antioquia, 60, 209-219.

Gómez, M. (2012). Los Informes de Responsabilidad Social Empresarial: su Evolución y Tendencias en el Contexto Colombiano. Cuadernos de Contabilidad, 13(32), 121-158.

Informe de la Comisión Brundtland. (1987). Comisión Mundial sobre Medio Ambiente y Desarrollo. Recuperado de http://www.un.org/es/comun/ docs/?symbol $=\mathrm{A} / 42 / 427$

Kotler, P. y Zaltman, G. (1971). Social marketing: an approach to planned social change. Journal of Marketing, 3(35), 3-12. 
Ministerio de Transporte. (2014). Mintransporte. Colombia. Recuperado de https://www.mintransporte.gov.co/Publicaciones/mintransporte_logra_ la_meta_de_ejecucion_mas_alta_de_toda_su_historia

Organization of the Pretroleum Exporting Countries. (2013). Indicadores del Mercado. Recuperado de http://www.opec.org/opec_web/en/data graphs/334.htm

Poder Público - Rama Legislativa Nacional. (Ley 454) (4 de agosto de 1998). República de Colombia, Diario Oficial, 43.357, 1998, 6, agosto.

\section{Para citar este artículo:}

Delgado, Y., Herrera, N., Gallón, C., Álvarez, M. y Muñoz, L. (2016). La Responsabilidad Social Empresarial, una mirada a su aplicación en el sector transporte público automotor. En-Contexto, 4(4), 77-99. 